\title{
Monohydric phenol impact on the state of cerebral blood flow, vegetative nervous system and cognitive functions of children
}

\author{
A. Aminova, Z. Gumbatova, S. Guseva \\ Sechenov First Moscow State Medical University, pediatric, Moscow, Russia.
}

Exposure to industrial toxicants is one of the stress factors that contribute to the stress of adaptation and compensatory mechanisms, and the disruption of activity of the autonomic nervous system. Phenol have a direct cytotoxic metabolic effects on the structures of central and peripheral nervous systems, and significantly contribute to the risk of development of neurological diseases and autonomic dysfunctions.

Materials and Methods. We used a complex of clinical and laboratorical (chemical and analytical) methods of examination and instrumental diagnostics to identify cognitive functions impairment, neuropsychological testing, including assessment of the overall maturity level, the memory storage capacity, the study of anxiety level. We conducted a comprehensive survey of 127 preschool-age children (5-7 years old) living in the condition where the average daily phenol concentration in the air. As a control group (unexposed), we examined 88 children living in the conventionally ecologically safe territory with levels of phenol in the blood that do not exceed the background values.

Results. The autonomic dysfunction associated with aerogenic phenol and cresol influence is characterized by initial vagotonia and tension of adaptive-compensatory mechanisms with excessive activation of humoral-metabolic segment under load (central regulatory mechanisms) and its subsequent depletion. It is an indicator of GW power at rest and orthostasis, and an index of vago-sympathetic balance in orthostasis (HW/SW). The linear velocity of the blood flow in the cerebral arteries, a low level of audio-verbal memory, the overall mental development and a high degree of anxiety are the effect markers of conditions of phenol and cresol influence. It is identified patterns of cognitive, behavioural status and cerebral haemodynamics are recorded on the background of marker deviations of homeostatic parameters concerning phenol in children with VSD in the form of increased level of regulatory hormones and cholinergic neurotransmitters (adrenocorticotropic hormone, cortisol, serotonin and (GMP), increased lipid peroxidation products, activation and consequent depletion of antioxidant blood activity and an increase of MDA concentration.

Conclusions. Submitted patterns of cerebral blood flow and cognitive and behavioural functions can be used as an additional criterion of dysfunctions of the central humoral-metabolic mechanisms of vegetative regulation in children with vegetative dystonia associated with influence of monohydric phenols.

\begin{tabular}{|c|c|c|}
\hline in blood & exposed, $(\boldsymbol{\mu g} / \mathbf{d L} \pm \mathbf{S E})$ & background level \\
\hline phenol & $0,045 \pm 0,002^{*}$ & $0,010 \pm 0,0012$ \\
\hline
\end{tabular}

\begin{tabular}{|l|c|c|c|c|c|}
\hline $\begin{array}{l}\text { in } \\
\text { blood }\end{array}$ & Increase probability & $\mathbf{F}$ & $\mathbf{p}$ & $\mathbf{R}^{\mathbf{2}}$ & $\mathbf{x}^{\mathbf{0}}$ \\
\hline Phenol & Nervous system diseases & 73,806 & $\mathbf{0 , 0 0 0 1}$ & 0,23 & 0,033 \\
\hline
\end{tabular}

$\mathrm{x} 0$ - no-effect level, $\mathrm{F}-\mathrm{F}$-criteria is used to check the significance of the multiple correlation index, R2 - determination index

Systolic/diastolic ratio (S/D) values in major cerebral arteries in the examined children.

\begin{tabular}{|l|l|l|l|l|}
\hline \multicolumn{2}{|l|}{ Major cerebral arteries } & Study group & Control group & P-value \\
\hline \multirow{2}{*}{ ACA } & Right & $2.78 \pm 0.52$ & $2.15 \pm 0.31$ & 0.003 \\
\cline { 2 - 5 } & Left & $2.54 \pm 0.48$ & $2.24 \pm 0.36$ & 0.039 \\
\hline \multirow{2}{*}{ MCA } & Right & $2.49 \pm 0.36$ & $2.09 \pm 0.20$ & 0.05 \\
\cline { 2 - 5 } & Left & $2.32 \pm 0.26$ & $1.89 \pm 0.16$ & 0.01 \\
\hline \multirow{2}{*}{ PCA } & Right & $2.67 \pm 0.58$ & $2.13 \pm 0.30$ & 0.014 \\
\cline { 2 - 5 } & Left & $2.67 \pm 0.44$ & $1.96 \pm 0.42$ & 0.01 \\
\hline
\end{tabular}

The increase of the phenol concentration in the air and level of diseases of the nervous system among children living in the area
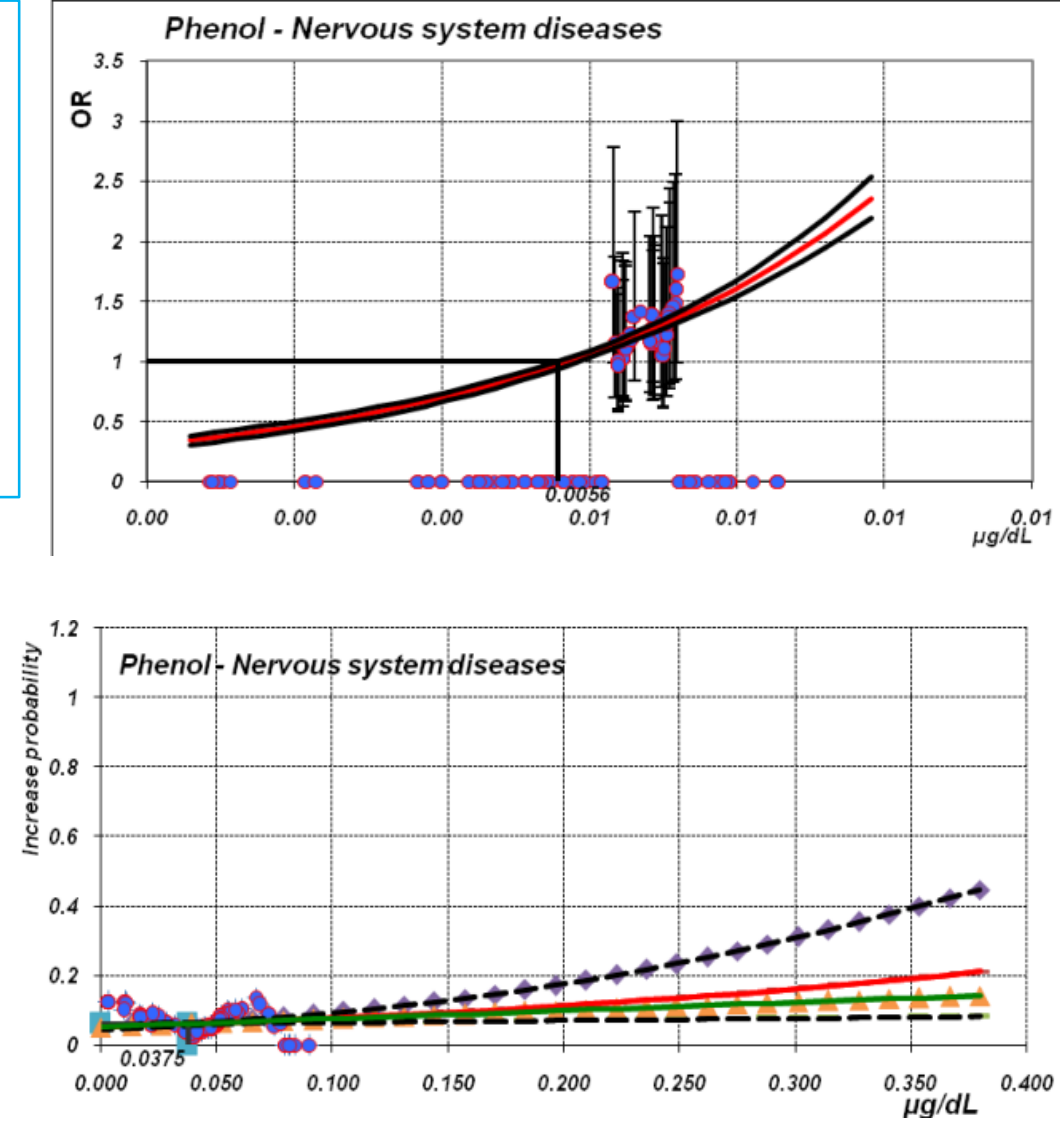

Mathematical model, reflecting the dynamics of increase of odds ratio index (OR) of formation of nervous system diseases in the conditions of the increasing phenol concentration in the air

Mathematical model reflecting the dynamics of increase of the probability of formation of nervous system disorders in the conditions of the increasing phenol concentration in blood. 